\title{
Horrifics of Pediatric Sedation: When Safety Guidelines Are Disregarded
}

\author{
John E Nathan*1-6 \\ ${ }^{1}$ Adjunct Professor, Department of Pediatric Dentistry, University of Alabama, Birmingham, and Case Western Reserve University, USA \\ ${ }^{2}$ Clinical Associate Professor, Department of Otolaryngology, Northwestern University Feinberg School of Medicine, USA \\ ${ }^{3}$ Diplomate, American Board of Pediatric Dentistry, USA \\ ${ }^{4}$ Fellow, American Academy of Pediatric Dentistry, USA \\ ${ }^{5}$ Fellow and Master, American Society of Dentistry for Children, USA \\ ${ }^{6}$ In private practice limited to Pediatric Dentistry, Oak Brook and St. Charles, USA
}

Submission: August 02, 2017; Published: August 04, 2017

*Corresponding author: John E Nathan, Adjunct Professor, Department of Pediatric Dentistry, University of Alabama, Birmingham, and Case Western Reserve University, Clinical Associate Professor, Department of Otolaryngology, Northwestern University Feinberg School of Medicine, Diplomate, American Board of Pediatric Dentistry, Fellow, American Academy of Pediatric Dentistry, Fellow and Master, American Society of Dentistry for Children, In private practice limited to Pediatric Dentistry, Oak Brook and St. Charles, USA, Email: jnathandds@gmail.com

\section{Editorial}

There is little that could constitute a more horrific occurrence than taking one's child for what should conceivably represent a routine dental visit only to experience an adverse and fatal reaction by virtue of an idiosyncratic, untoward, or irresponsibly managed sedation or anesthetic technique. Regrettably, this scenario has been reported numerous times over the past two decades as result of the use of some form of sedation or anesthesia intended to calm a child and aid in their acceptance of care.

Dental treatment can represent a particularly threatening event for some children; despite such hundreds of thousands of children, however, undergo routine repair of dental caries on a daily basis throughout the U.S.

Most possess cooperative potential to enable care to be rendered using conventional communication strategies without need for pharmacological intervention. For these, a soft voice and gentle demeanor are all that is needed to overcome mild anxiety to secure patient acceptance. For others, apprehension levels and behavioral resistance can be sufficiently profound that conventional standard techniques prove inadequate or inappropriate [1,2]. Dentists and in particular pediatric specialists have a variety of (alternative) pharmacological strategies in their armamentarium, inclusive of physical restraint for managing challenging and interfering child behavior [3]. While some clinicians are not well versed with sedative techniques, the majority of clinicians (and parents) appear to prefer use of a pharmacologic approach to the application of restraints to overcome disruptive or interfering child behaviors $[4]$.
Those proficient in the selection and deployment of various levels of sedation safely provide needed care to children lacking cooperative potential daily without incident or catastrophic outcomes. Accompanying these modalities are risks that are real and emphasize the essential need to comply with existing safety guidelines. Based on the severity of apprehension, the relative invasiveness of the task, and the duration of cooperation required, clinicians are required to exercise considerable judgment when choosing agents and dosing to meet the needs of their particular patient. Stimulus for this manuscript is the recurring nature by which compliance with safety standards appears lacking and sedation fatalities occur.

Despite great experience, a thorough understanding of the existing sedation literature, comprehensive knowledge and experience using all levels of sedation, and a better command of the nuances and subtleties observing child behaviors, even talented clinicians regard agent and dosage selection as much an art as science $[4,5]$. Selection of a pharmacological approach that carries the capacity to depress consciousness and/or protective reflexes leaves little room for error or a lack of proficiency in one's ability to recognize and manage an adverse reaction or medical emergency. To best comprehend the complexities and relative risks associated with the use of sedation, an appropriate starting point is to understand the various levels of sedation within the sedation arsenal.

The differing levels of sedation include "mild sedation" (nitrous oxide-oxygen, with or without minimal dosages of single agent sedatives such as hydroxyzine. Mild sedation minimally depresses consciousness and patients remain fully conscious 
and responsive to verbal communication AT ALL TIMES "Moderate sedation" intended when behavioral resistance and anxiety mandate implies a deeper level of CNS depression where patients may close their eyes, appear to become somnolent at some points of a visit, but upon minimal verbal or physical stimulation, will immediately re-open their eyes and respond appropriately. At no time, do vital signs or protective reflexes show evidence of compromise. Restricting sedations to mild or moderate levels should virtually never result in untoward reactions. Exceptions can however occur under circumstances where airways can be compromised by emesis or foreign body.

A level of depression that manifests somnolence and need for noxious physical stimulation to cause patient arousal is termed "Deep Sedation, "used when moderate sedation proves insufficient. This is a volatile if not potentially dangerous level of depression in a non-operating room environment. While differentiations of these three levels are easily determined, inductions of deeper planes of sedation intentionally or inadvertently readily occur in pediatric, adolescent, and adult sedation. It is in this level of sedation that adverse reactions with catastrophic consequences occur. Patient responses to varying dosages of sedative agents or combination of agents can be highly variable and it is in this arena that adverse reactions and need for prompt and proficient management occur. In most pediatric practice settings, the level of depression sought is limited to Moderate Sedation. That said, there are no guarantees that dosages of agents produce universal effects on all children or adults, and preparedness for induction of deeper than intended levels of depressed consciousness can unexpectedly occur at any time.

Practitioners must be proficient in recognizing alterations, subtle, or overt, in patient responses to agents they deploy. Responsibilities for patient monitoring have been well established since 1985 with updated revisions to further enhance dentist and patient safety occurring on an ongoing annual basis. Induction of deeper levels of sedation is entirely unintentional. Intentional induction of deep sedation, or General Anesthesia, in which surgical planes of anesthesia are produced requires administration by personnel formally trained in the field of anesthesiology within properly equipped facilities. Formal guidelines, developed by numerous societies (American Academy of Pediatric Dentistry, American Academy of Pediatrics, American Society of Anesthesiology, and others) both independently and collaboratively represent the standard of care. Despite such, numerous reasons account for the continued occurrence of incidences of morbidity and mortality. These will be described below.

Recently, a segment of Sunday Night with Megyn Kelly (airing July, 9, 2017) offered a critical look at horrific outcomes which continue to occur when sedation and general anesthetics are deployed by dentists and oral and maxillofacial surgeons for some children presenting with dental decay, invasive treatment needs, or impacted third molars. Understandably, this is unsettling for practitioners who by virtue of proficiency, diverse experience, and expanded knowledge safely perform sedation and general anesthetics daily without consequence or hazardous outcome. On the other hand, it is recognized that the media has responsibility to protect the public by any means if it suspects regulation or its oversights have serious flaws in self-assessment of practitioners lacking proficiency in their knowledge or compliance with known guidelines to ensure patient safety.

The focus of this 11 minute documentary was to alert the public to ask relevant questions of providers of care making use of sedative agents regarding safety measures they employ and preparedness to prevent, recognize, and manage a medical complication should one arise. While incidents were superficially presented, specifics related to the origin of mishaps intentionally excluded or kept vague, the message was clear. Can regulatory agencies and various involved disciplines do more to prevent these horrific outcomes? Better understanding of the causes of untoward reactions and adverse outcomes offers insights as to what might be done to reduce or eliminate their occurrence.

\section{Causes of untoward reactions and adverse outcomes}

Several sources have attempted to address the most common of causes of morbidity and mortality

from pediatric sedation [6-8]. They include but are not limited to:

a. Lack of familiarity with agents, their pharmacological and physiologic effects, and proper administration and dosing

b. Failure to perform or secure Review of Systems, physical evaluation of the patient pre-drug administration, post-drug administration, intra-operatively, post-operatively, and to ensure fulfillment of discharge criteria and post-sedation management

c. Failure to make use of appropriate agent(s) or combinations using responsible dosing

d. Failure to monitor the patient as per the needs dictated by the level of depression secured (vital signs, oxygen perfusion, ventilation or capnography, protective reflexes, airway patency, etc.

e. Insufficient knowledge of toxic dosage parameters of local anesthetic and rate of administration.

f. Inadequacy of the facility equipment and emergency armamentarium and proficiency of its personnel in their ability to recognize and manage a medical emergency.

g. Failure to understand the duration of action of many long-acting sedative agents which carry active metabolites and their potential for adversity at and after discharge. 
h. Failure to have an existing emergency protocol in place for all personnel with periodic evidence to verify proficiency and performance.

i. Unrealistic expectations for the indications, benefits, shortcomings of the modality chosen.

Upon analysis of the etiology of almost every tragedy involving sedation, one or more of these generic etiologies apply. Only rarely has idiosyncratic or undiagnosed cardiovascular anomalies been found to serve as a cause. The American Academy of Pediatric Dentistry has stated that no incidents of fatality have occurred when compliance with published safety guidelines have occurred.

At present, continuing education courses are appearing which offer weekend instruction for the purpose of advocating how to safely sedate children and adult patients. Attraction on the basis of increased profitability is a core element for these endeavors. Whether these courses offer sufficient coverage to enable safe and efficacious care given limited time commitments and hands-on experience remains unclear. It seems plausible that experienced clinicians with considerable expertise in advanced airway and emergency medical management will find these formats for sedation helpful to refine their skills. Skepticism however prevails as to the viability and safety of such for the novice seeking to add sedation to their practice. Currently no regulation appears on the horizon for this category of continuing education [9].

To date, questions remaining turn toward what additional measures can be taken by regulatory agencies, state dental commissions, and accreditation Bodies and University advanced training programs to reduce morbidity and mortality amongst children undergoing sedation? Do states have the resources to monitor and assess practitioner proficiency and facility preparedness? Alternatively are actions forthcoming only following an incident that comes to the public's attention from negligence or malpractice?

Can dentistry disciplines such as the American Academy of Pediatric Dentistry whose focus is the well-being of children, take on a more proactive role? No doubt, political interests play a role in policy of an organization claiming to be the experts in child dental health, and in particular under circumstances in which morbidity and fatality resulting in litigation ensues. A logical deterrent to reduce the incidence of pediatric incidents might be to restrict, legislate, or more closely scrutinize in-office sedations to the use of mild or moderate sedation. Only those including administration and monitoring by a licensed and trained anesthesiologist can make use of deep sedation in an office setting.

No data bank currently records relevant details of instances involving morbidity and mortality. The dental societies appear to not have willingness to intervene by amassing a collection of detail to illustrate shortcomings and serve as a teaching tool to deter and intercept potential tragedies. Precedent exists regarding a national data bank that identifies any physician or dentist who has lost privileges or appointment at a hospital for a wrongdoing. What harm could come from the formulation of a national data bank of childhood catastrophic outcomes, assuming all rights of privacy are preserved? [10]. When will we see a stop to these horrific occurrences?

\section{References}

1. Nathan JE (2016) The Evolution and Changing Patterns of Behavioral Management of Challenging Childhood Dental Anxiety: A Crossroad. Dentistry 6(5): 1-2.

2. Nathan JE (2017) Helping Anxious and Uncooperative Children to cope with Invasive Dental Care using Sedation: A Double-Edged Sword. Oral Health and Dentistry 1(2): 104-106.

3. Nathan JE (2017) The Appropriateness (or lack thereof) of Physical restraints for Managing Challenging Pediatric Dental Behavior. J Pediatrics and Neonatal Nursing 4(1): 1-3.

4. Nathan JE (2015) The Direction of Pediatric Sedation: A Contemporary Look at it Science, Art, Strengths, and Shortcomings. Inter J of Otolaryngology 2(1): 8-15.

5. Nathan JE (2015) The Subtleties and Nuances of Pediatric Sedation: A Disappearing Art for managing the Apprehensive Child Patient. J of Otolaryngology and Rhinology 1(1): 1-2.

6. Cote CJ, Karl HW (2000) Adverse Sedation Events in Pediatrics: Analysis of Medications usedin Sedation. Pediatrics 106: 633-644.

7. Nathan JE (2016) Morbidity and Mortality involving Pediatric Sedation: Non-compliance following Sedation Safety Guidelines. Journal of Surgery 1(1): 1-3.

8. Cravero JP (2006) Incidence and Nature of Adverse Events during Pediatric Sedation for Procedures outside the Operating Room, Report from Pediatric Sedation Research Consortium. Pediatrics 118: 10821096.

9. Nathan JE (2017) Pediatric sedation; A Predictable and Practical Approach to Avoid Mishaps, Adverse Reactions and Catastrophic Outcomes. J Pharmacology and Clinical Research 2(4): 1-3.

10. Nathan JE (2017) What will it take to prevent another Pediatric Sedation or Anesthetic Fatality. Dentistry 7(7): 122. 

(C) This work is licensed under Creative

DOI: 10.19080/OAJS.2017.05.555655

\section{Your next submission with Juniper Publishers} will reach you the below assets

- Quality Editorial service

- Swift Peer Review

- Reprints availability

- E-prints Service

- Manuscript Podcast for convenient understanding

- Global attainment for your research

- Manuscript accessibility in different formats

( Pdf, E-pub, Full Text, Audio)

- Unceasing customer service

Track the below URL for one-step submission https://juniperpublishers.com/online-submission.php 